\title{
Centralização do Fluxo Sangüineo Fetal Diagnosticado pela Dopplervelocimetria em Cores: Resultados Perinatais
}

\author{
Brain-sparing Effect Detected by Color Doppler: Perinatal Outcome \\ Cleide Mara Mazzotti Oliveira Franzin, João Luiz Pinto e Silva, \\ Emílio Francisco Marussi, Silvana Varella Parmigiani
}

\begin{abstract}
RESUMO
Objetivos: avaliar os resultados perinatais do exame de dopplervelocimetria alterado com centralização de fluxo sangüineo fetal.

Metodologia: foram analisados 32 casos de centralização de fluxo sangüineo fetal diagnosticados no Centro de Atenção Integral à Saúde da Mulher da Universidade Estadual de Campinas. O diagnóstico de centralização foi confirmado quando a relação entre o índice de pulsatilidade da artéria cerebral média fetal (IPACM) e o indice de pulsatilidade da artéria umbilical (IPAU) era menor que a unidade (IPACM/IPAU menor que 1). Detectou-se fluxo arterial usando equipamento ultra-sonográfico equipado com mapeamento em cores, marca Toshiba, modelo SSH-140A.

Resultados: houve necessidade de cuidados intensivos em 26 fetos (89,6\%). O número de dias de internação em unidade de terapia intensiva variou de 1 a 83, com média de 22 dias. A ocorrência de óbito fetal foi de 3/32 (9,4\%) e de óbito perinatal de 9/29 (31\%). Considerando-se a idade gestacional avaliada pelo método de Capurro, a incidência de nascimento de fetos com menos de 36 semanas foi de 21/32 (65,6\%). Crescimento intrauterino restrito ocorreu em $71,8 \%$ dos fetos e hipoglicemia em $44,8 \%$.

Conclusão: a centralização de fluxo sangüineo é um marcador de situação danosa ao bemestar fetal e seu estudo será de grande valia na orientação da conduta obstétrica.
\end{abstract}

PALAVRAS-CHAVE: Dopplervelocimetria. Centralização de fluxo sangüineo. Morbidade perinatal.

\section{Introdução}

A ultra-sonografia, como método propedêutico, revolucionou a prática obstétrica, possibilitando acesso a informações até então desconhecidas, que facilitaram muito o desempenho clínico. O aperfeiçoamento e a introdução do efeito Doppler aos equipamentos ultra-sonográficos propiciaram estudo in vivo das circulações fetoplacentária e útero-placentária, permitindo avaliar o grau de resistência ao fluxo sangüíneo e sua Departamento de Tocoginecologia da Faculdade de Ciências Médicas da Universidade Estadual de Campinas (UNICAMP).

Correspondência

Cleide M. M. Franzin

A/C - Assessoria Técnica e Científica do CAISM

Rua Alexander Fleming, 101 - Cidade Universitária "Zeferino

Vaz"

13083-970 - Campinas - SP relação com o estado de saúde do concepto.

Antes do advento da ultra-sonografia, os conhecimentos de anatomia e fisiologia da circulação feto-placentária eram obtidos por meio de estudos invasivos com animais ${ }^{1,2}$, pela placentografia com rádioisótopos ${ }^{3}$ ou estudos com óxido nitroso para avaliar o fluxo sangüíneo na placenta humana $^{4}$. FitzGerald e Drumm ${ }^{5}$ realizaram os primeiros registros de fluxo sangüíneo com Doppler pulsátil em Obstetrícia ${ }^{5}$.

Utilizando-se o mecanismo Doppler, obtémse a análise do perfil de velocidade de fluxo, refletindo as condições circulatórias nas direções anterógrada e retrógrada ${ }^{6}$ Para avaliar a resistência vascular periférica, são utilizados vários indices, denominados indice de resistência ${ }^{7}$, indice de pulsatilidade ${ }^{8}$ e relação sístole/diástole ${ }^{9}$.

Com a evolução da gestação normal, ocorre diminuição da resistência ao fluxo sangüineo na 
circulação umbilical e aumento progressivo no componente diastólico final ${ }^{10}$ em decorrência da proliferação do sistema viloso terciário, ampliando, portanto, as áreas de trocas nas vilosidades ${ }^{11}$.

Usando estes conhecimentos, vários pesquisadores começaram a relatar a possibilidade de avaliar alterações na circulação útero-placentária associadas às patologias fetais. Constatou-se associação entre a redução do componente diastólico final da artéria umbilical e, portanto, aumento da resistência vascular, com retardo de crescimento intra-uterino e aumento da mortalidade perinatal $^{12}$.

O fenômeno de centralização fetal (brainsparing effect) é um mecanismo conhecido desde o final da década de $60^{13-15}$, mediante estudos com animais. Constatou-se que a hipoxemia fetal estava associada à redistribuição hemodinâmica do fluxo sangüíneo, resultando em perfusão preferencial do sangue para órgãos nobres, tais como: cérebro, coração e glândulas adrenais, em detrimento da perfusão de pulmão, rins, baço e esqueleto, ou seja, havia vasoconstrição periférica e vasodilatação cerebral.

Posteriormente constatou-se que os fetos humanos que acionavam este mecanismo de defesa apresentavam maior risco de mortalidade e morbidade perinatais.

Wladimiroff et al. ${ }^{16}$ introduziram o estudo do fluxo sangüíneo cerebral com o Doppler, constatando que em fetos com crescimento intra-uterino restrito (CIUR), o indice de pulsatilidade (IP) da artéria carótida interna apresentava-se reduzido (com vasodilatação), embora estivesse aumentado na artéria umbilical e aorta torácica, sugerindo aumento da resistência vascular placentária e periférica fetal, com redução compensatória da resistência ao fluxo sangüíneo cerebral .

O diagnóstico da centralização pode ser realizado pela relação entre o IP da artéria umbilical e o IP da artéria cerebral média (IPAU/IPACM), que reflete, melhor que o estudo de um único vaso, as modificações hemodinâmicas que ocorrem durante o fenômeno de "preservação cerebral"17.

Tais estudos foram confirmados com a relação inversa, ou seja, com a relação entre o IP da artéria cerebral média e o IP da artéria umbilical. Todos os valores superiores a 1,08 entre a $3^{\mathrm{a}}$ e a $41^{\text {a }}$ semanas foram considerados normais. Para resultados perinatais adversos a acurácia diagnóstica desta relação foi de $90 \%$, comparada com $78,8 \%$ e $83,3 \%$ para as artérias cerebral média e umbilical, respectivamente ${ }^{18}$.

Para determinar a acurácia da relação entre o IR (indice de resistência) da artéria cerebral média e o IR da artéria umbilical, na predição de prognóstico fetal, Arias, em $1994{ }^{19}$, estudou paci- entes cujo exame ultra-sonográfico tinha sido realizado até duas semanas antes do nascimento. Concluiu que a relação menor ou igual à unidade identificava o alto risco para CIUR e para morbidade neonatal grave, sugerindo ser inadequada a avaliação de fetos apenas com o Doppler umbilical, e que se poderia obter melhor indicador do prognóstico fetal pela avaliação conjunta da circulação umbilical e cerebral.

A redistribuição sangüínea que ocorre em fetos com restrição de crescimento intra-uterino é regulada por mais de um mecanismo. A hipercapnia isoladamente, ou associada à acidemia, desempenha um papel de controle das respostas vasculares da carótida e aorta, ao passo que a hipoxemia isoladamente, ou por meio da hipercapnia, seria responsável pela resposta vascular cerebral ${ }^{20}$.

Estudos recentes têm confirmado a associação significativa entre a centralização sangüínea fetal e o aumento da mortalidade e morbidade em fetos com restrição de crescimento com menos de 34 semanas de gestação, sugerindo que não haveria associação com resultados adversos após a $34^{\mathrm{a}}$ semana $^{21}$.

A proposta deste trabalho é analisar a repercussão fetal e neonatal da doppervelocimetria alterada com centralização de fluxo sangüíneo fetal, e contribuir para estabelecer sua validade clínica.

\section{Pacientes e Métodos}

Foram analisados retrospectivamente 32 casos de centralização de fluxo sangüíneo fetal, diagnosticados no Setor de Ultra-Sonografia do Centro de Atenção Integral à Saúde da Mulher (CAISM) da Universidade Estadual de Campinas (UNICAMP). Fizeram parte do estudo pacientes grávidas com centralização de fluxo sangüíneo fetal, atendidas entre fevereiro de 1995 e novembro de 1997, selecionadas pelo banco de dados do setor de ultra-sonografia. O diagnóstico de centralização foi realizado por meio da análise da relação entre o IP da artéria cerebral média e o da artéria umbilical, considerando-se anormais valores menores que a unidade (IPACM/IPAU menor que um). Para os casos com diástole ausente ou reversa na artéria umbilical, utilizamos a relação entre o IR da artéria cerebral média e o da artéria umbilical menor que a unidade (IRACM/ IRAU menor que um).

Foram excluídas as gestações gemelares, as com malformações fetais e com doenças auto-imu- 
nes fetais. Excluímos também as pacientes cujo parto não ocorreu nesta instituição.

A faixa etária das gestantes variou de 15 a 44 anos, com valor médio de 29 anos. A média do número de gestações foi de 3,2 , com média de paridade de 1,6. A análise da paridade revelou que $18 \%$ das pacientes estavam grávidas pela primeira vez e $31 \%$ tiveram de 1 a 3 partos anteriores.

A maioria das gestantes foi considerada de alto risco (condição clínica que pudesse representar algum dano para a saúde materno fetal), sendo as doenças mais freqüentes: DHEG (doença hipertensiva específica da gestação) - 18 casos $(56,2 \%)$ e hipertensão arterial crônica - 6 casos $(18,7 \%)$. Houve um caso de diabetes gestacional e um de amniorrexe prematura (Tabela 1). Não apresentaram doença aparente seis gestantes, sendo a indicação do exame para elas a suspeita clínica de restrição de crescimento intra-uterino ou oligoâmnio.

Tabela 1 - Doenças maternas dos 32 casos de centralização.

\begin{tabular}{lcr}
\hline & $\mathbf{n}$ & \% \\
\hline DHEG & 15 & 46,8 \\
DHEG + ca de colo & 1 & 3,1 \\
DHEG + síndrome HELLP & 1 & 3,1 \\
DHEG + hipertiroidismo & 1 & 3,1 \\
HAC & 4 & 12,5 \\
HAC + aneurisma cerebral & 1 & 3,1 \\
HAC + HIV & 1 & 3,1 \\
Diabetes gestacional & 1 & 3,1 \\
Amniorrexe prematura & 1 & 3,1 \\
Sem patologia & 6 & 18,7 \\
\hline
\end{tabular}

DHEG, doença hipertensiva específica da gestação;

HELLP, "hypertension elevated liver enzymes low platelet counts";

HAC, hipertensão arterial crônica; HIV, "human immunodeficiency virus".

À época da interrupção da gravidez, a idade gestacional variou de 24 a 39 semanas. A proporção de recém-nascidos com menos de 36 semanas de gestação foi de 21/32 (65,6\%) (Figura 1).

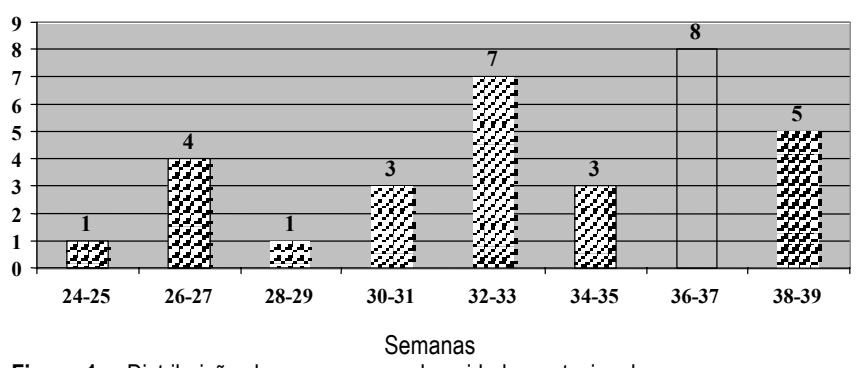

Figura 1 - Distribuição dos casos segundo a idade gestacional.
Analisamos os seguintes resultados neonatais: idade gestacional, peso do concepto, adequação do peso à idade gestacional, mortalidade fetal, mortalidade perinatal, indices de Apgar $(1953)^{22}$ de $1^{\circ}$ e $5^{\circ}$ minuto, necessidade de internação em unidade de terapia intensiva e morbidade fetal avaliada através da presença de: hipoglicemia, membrana hialina, septicemia, hemorragia cerebral, hemorragia pulmonar e enterocolite necrosante. Analisamos também a microscopia placentária.

A idade gestacional foi calculada pelo indice somático de Capurro et al. ${ }^{23}$. Nos fetos com morte intra-uterina a idade gestacional foi avaliada por meio da ultra-sonografia mais precoce, utilizando-se tabelas de biometria fetal desenvolvidas no setor ${ }^{24}$.

Restrição de crescimento intra-uterino foi definido como peso inferior ao percentil 10 para a idade gestacional, de acordo com a curva de Hadlock et al. ${ }^{25}$. Para o diagnóstico pós-natal, utilizamos a curva de adequação de peso de Lubchenco et al. ${ }^{26}$, adotada pelo Serviço de Neonatologia do CAISM.

Para o diagnóstico de hipoglicemia, utilizamos os seguintes parâmetros de normalidade maior ou igual a $25 \mathrm{mg} \%$ (para o periodo de 28 a 36,6 semanas) e maior ou igual a $30 \mathrm{mg} \%$ (acima de 37 semanas de gestação), padronizados pelo serviço.

A hemorragia cerebral foi diagnosticada pela presença de imagem hipoecóica, sugestiva de hemorragia peri ou intraventricular, visualizada por meio de ultra-sonografia transfontanela.

A microscopia e a macroscopia placentária foram realizadas pelo Departamento de Anatomia Patológica da UNICAMP.

A dopplervelocimetria foi realizada com equipamento marca Toshiba, modelo SSH-140 A. Obteve-se fluxo arterial usando equipamento de Doppler duplex, com mapeamento em cores, com transdutor convexo de 3,75 MHz. Após boa audição e visualização dos sinais, a imagem foi congelada, realizando-se planigrafia da onda com calibradores eletrônicos. Após ajuste adequado do ângulo entre o cursor e o vaso, as medidas de velocidade sistólica, diastólica e média foram fornecidas automaticamente pelo equipamento.

A potência acústica do modo Doppler com mapeamento em cores manteve-se abaixo dos padrões estabelecidos pela Food and Drug Administration (FDA) em 1976 e pela Federation International Gynecology and Obstetrics (FIGO) em $1991^{27}$ para a ultrasonografia fetal, com intensidade média do pico espacial temporal de $57 \mathrm{~mW} / \mathrm{cm}^{2}$.

Após visualização da artéria umbilical com mapeamento em cores, colocou-se o cursor no lúmen do vaso que se apresentava paralelo à onda 
ultra-sônica, com ângulo de incidência inferior a 30 graus. Foram realizadas medidas longe da inserção placentária e fetal ${ }^{28}$.

Para a localização da artéria cerebral média, primeiramente obteve-se corte transversal do pólo cefálico, incluindo o tálamo e o cavum do septo pelúcido (plano em que o diâmetro biparietal é medido). A seguir, movimentando o transdutor caudalmente, no plano paralelo ao plano previamente tomado, identificou-se a asa maior do osso esfenóide entre as fossas média e anterior ${ }^{29}$. Usouse o mapeamento em cores para identificar o polígono de Willis e a artéria cerebral média foi facilmente identificada como o maior ramo ânterolateral do polígono, dirigindo-se à borda lateral da órbita. Posicionou-se o cursor no lúmen da artéria cerebral média próxima ao transdutor, no trecho compreendido entre $1 \mathrm{~cm}$ da sua origem na artéria carótida interna ${ }^{19}$. O ângulo entre o cursor e o vaso não excedeu 30 graus.

Os resultados obtidos neste estudo foram apresentados em números absolutos e percentuais, dispensando análise estatística mais acurada devido ao pequeno número de casos.

A conduta obstétrica preconizada para a decisão final considerou os diversos recursos propedêuticos utilizados no serviço, após discussão em conjunto.

\section{Resultados}

O peso ao nascimento variou de $520 \mathrm{~g}$ a 3.320 $\mathrm{g}$, com valor médio de $1.420 \mathrm{~g}$. Peso inferior a 1.000 $\mathrm{g}$ foi observado em 10 crianças, sendo que oito apresentaram peso entre $500 \mathrm{~g}$ e $750 \mathrm{~g}$.

A corticoterapia profilática foi ministrada para $62,5 \%$ das pacientes, fazendo parte da rotina do serviço. As mulheres que não receberam o tratamento profilático geralmente foram encaminhadas de outras instituições, não havendo tempo hábil para sua realização, considerando a gravidade do caso.

A maioria dos recém-natos $(89,6 \%)$ necessitou de cuidados em unidade de terapia intensiva (Tabela 2). O número total de dias de internação variou de 1 a 83, com média de 22 dias. Óbito intrauterino ocorreu em 3 casos $(9,4 \%)$. Estes fetos tinham peso ao nascimento entre $520 \mathrm{~g}$ e $580 \mathrm{~g}$, e apresentaram diástole zero ou reversa na artéria umbilical. A ocorrência de óbito pós-natal foi de $31 \%$ (9/29). A análise destes casos de óbito pósnatal mostrou que sete apresentaram componente diastólico ausente ou reverso na artéria umbilical e peso entre $500 \mathrm{~g}$ e $750 \mathrm{~g}$. Dois fetos apresentaram peso acima de $2.500 \mathrm{~g}$.
Tabela 2 - Resultados perinatais adversos dos 32 casos de centralização.

\begin{tabular}{lrr}
\hline & $\mathbf{n}$ & $\%$ \\
\hline Internação em UTI & 26 & 89,6 \\
Óbito fetal & 3 & 9,4 \\
Óbito perinatal & 9 & 31,0 \\
CIUR & 23 & 71,8 \\
Hipoglicemia & 13 & 44,8 \\
Membrana hialina & 4 & 13,8 \\
Septicemia & 5 & 17,2 \\
Hemorragia cerebral & 2 & 6,9 \\
Hemorragia pulmonar & 2 & 6,9 \\
\hline
\end{tabular}

CIUR, crescimento intra-uterino restrito.

Ao nascimento, o valor do índice de Apgar do $1^{\circ}$ minuto foi menor que sete em 10 casos $(34,5 \%)$. Índice de Apgar do $5^{\circ}$ minuto menor que sete ocorreu em dois casos $(6,8 \%)$.

Ocorreu restrito de crescimento intrauterino em $71,8 \%$ dos fetos e hipoglicemia em $44,8 \%$ (Tabela 2). Outras intercorrências mais freqüentes no período pós-natal foram: membrana hialina $(13,8 \%)$, septicemia $(17,2 \%)$, hemorragia cerebral, hemorragia pulmonar, policitemia e íleo prematuro ( 2 casos cada). Neste estudo ocorreu apenas um caso de enterocolite necrosante, com recém-nascido pesando 1.030 $\mathrm{g}$ ao nascimento. Evoluiu com rotura intestinal, com ressecção de segmento de cólon transverso e íleo terminal, necessitando de cuidados intensivos durante 83 dias. Ao Doppler apresentava diástole reversa na artéria umbilical. Teve alta em bom estado.

Não foi possível a obtenção de exame microscópico em todas as placentas. Em apenas três casos o resultado anatomopatológico foi normal. Áreas de infarto placentário foram diagnosticadas em 13 casos (40,6\%). Detectaram-se também: cisto septal, congestão intensa, hematoma de seio marginal, autólise extensa e vilosite crônica (Tabela 3).

Tabela 3 - Microscopia placentária de alguns casos de centralização.

\begin{tabular}{lrr}
\hline Resultado & $\mathbf{n}$ & $\%$ \\
\hline Normal & 3 & 9,4 \\
Infarto & 13 & 40,6 \\
Cisto septal & 1 & 3,1 \\
Congestão intensa & 1 & 3,1 \\
Vilosite crônica & 1 & 3,1 \\
Hematoma do seio marginal & 1 & 3,1 \\
Autólise extensa & 1 & 3,1 \\
\hline
\end{tabular}




\section{Discussão}

Analisando a mortalidade neonatal geral em nosso Serviço, entre fevereiro de 1995 a novembro de 1997, observamos que para fetos com peso ao nascimento entre $500 \mathrm{~g}$ e $749 \mathrm{~g}$, a taxa de mortalidade foi de $70 \%$; entre $750 \mathrm{~g}$ e $999 \mathrm{~g}, 35 \%$; entre $1.000 \mathrm{~g}$ e $1.499 \mathrm{~g}, 14 \%$, diminuindo progressivamente; entre $2.500 \mathrm{~g}$ e $2.999 \mathrm{~g}, 2,5 \%$, e acima de 3.000 g, 0,6\% de mortalidade. Em 32 casos de centralização de fluxo analisados, a mortalidade neonatal foi de $28 \%$. Na faixa de peso entre $500 \mathrm{~g}$ e $749 \mathrm{~g}$, a mortalidade foi de $87 \%$; entre $2.500 \mathrm{~g}$ e $2.999 \mathrm{~g}$, foi de $66 \%$, maiores, portanto, que a taxa obtida para a população geral.

Constatamos que neste grupo de fetos "centralizados", aqueles com componente diastólico ausente ou reverso apresentaram maior morbidade, pois entre os nove recém-nascidos que morreram, sete apresentaram componente diastólico ausente ou reverso na artéria umbilical.

A presença de diástole zero ou reversa na artéria umbilical indica parada do fluxo sangüíneo para frente em uma parte de cada ciclo cardíaco, refletindo o grau máximo de alteração do Doppler na artéria umbilical. Comparando-se os resultados perinatais dos fetos com centralização de fluxo com estudo anterior em fetos com diástole zero ou reversa ${ }^{30}$, constatamos resultados piores neste último, com 93,3\% de restrição de crescimento, $43,3 \%$ de mortalidade perinatal e $100 \%$ de necessidade de cuidados em unidade de terapia intensiva.

Apesar dos sérios distúrbios hemodinâmicos, observamos que o indice de Apgar do $5^{\circ}$ minuto, em média, foi maior que sete. Alguns pesquisadores ${ }^{31}$ ignoram o Apgar do $5^{\circ}$ minuto por considerá-lo inadequado no diagnóstico de hipóxia neonatal, pois somente $15 \%$ dos recém-nascidos afetados por paralisia cerebral têm índices de Apgar baixos. Sykes et al. ${ }^{32}$ também encontraram fraca correlação entre índices de Apgar e valores de $\mathrm{pH}$ umbilical.

Neste estudo, apesar do grande número de prematuros, a incidência de doença da membrana hialina mostrou-se relativamente baixa, provavelmente em decorrência de hipoxemia crônica e pelo uso de corticoterapia antenatal sistemática para estimular a maturação pulmonar fetal.

A morbidade dos fetos com centralização de fluxo sangüíneo é considerável. Constatou-se neste estudo alta incidência de necessidade de internação em UTI neonatal por periodos prolongados, com média de internação de 22 dias e grande incidência de doenças fetais graves, tais como hemorragias pulmonar cerebral e enterocolite necrosante.
Montenegro et al. ${ }^{33}$, classificaram a história natural da centralização em três estágios: centralização normoxêmica, hipoxêmica e descentralização. Na centralização normoxêmica, o sangue coletado por meio de cordocentese exibe acidemia, mas com niveis de $\mathrm{PO}_{2}$ normal-baixo, pois ocorre acúmulo de ácido lático produzido pela respiração anaeróbica nos territórios que apresentam vasoconstrição e deficiente aporte de $\mathrm{O}_{2}$. Neste estágio, a cardiotocografia basal ainda é reativa, pois o coração e o cérebro têm oxigenação preferencial. Na centralização hipoxêmica, a hipoxemia fetal se agrava e a redistribuição sangüínea não assegura normoxemia ao cérebro e ao coração. Neste estágio, o feto apresenta hipoxemia e acidemia. Quando a redistribuição do fluxo sangüíneo entra em colapso pela descompensação cardíaca e pelo edema cerebral, a cordocentese aponta hipoxemia, acidemia extrema e hipercapnia.

Os conhecimentos dos fatores de ajuste circulatório e seu mecanismo de ação são incompletos. Provavelmente quando a pressão parcial de $\mathrm{O}_{2}$ diminui e a de $\mathrm{CO}_{2}$ se eleva acima de um certo nível, os quimiorreceptores aórticos e carotídeos podem ser ativados, regulando a resposta vasodilatadora central com a finalidade de garantir adequada oxigenação ao cérebro fetal ${ }^{34}$.

Constatou-se neste estudo grande número de alterações placentárias. O Doppler alterado da artéria umbilical foi correlacionado, pela primeira vez, com anatomia microvascular placentária por Giles et al. ${ }^{35}$, em que a resistência arterial placentária foi quantificada pelo número de pequenas artérias no sistema viloso terciário. No grupo com relação sístole/diástole aumentada, o número de pequenos vasos arteriais foi significativamente menor (1-2 artérias por campo) do que no grupo normal (7-8 artérias por campo).

Alguns pesquisadores utilizam apenas a artéria cerebral média alterada para identificação de centralização sangüínea fetal. Acreditamos, assim como outros autores, que o diagnóstico pela relação IPACM/IPAU reflete melhor que um único vaso as alterações hemodinâmicas da preservação cerebral. O Doppler alterado na artéria cerebral está associado com pior prognóstico fetal. Mari e Deter ${ }^{29}$ constataram $60 \%$ de sensibilidade e $87 \%$ de especificidade da artéria cerebral média alterada, para resultados perinatais adversos. Todavia, neste estudo, três crianças com fluxo cerebral normal morreram, sendo uma intra-útero e duas logo após o nascimento. Observaram que o IP da artéria cerebral média é mais baixo entre a $15^{\mathrm{a}}$ e $20^{\mathrm{a}}$ semana e no final da gestação, indicando diminuição da resistência vascular cerebral ao fluxo sangüíneo nestes dois períodos de gestação. 
Dobbing e Sands ${ }^{36}$ estudaram cérebros fetais humanos e estimaram o número total de células, por análise química de ácido desoxirribonucléico no cerebelo, tronco e porção anterior do cérebro. Demonstraram haver dois periodos de maior multiplicação celular, da $15^{\mathrm{a}}$ à $20^{\mathrm{a}}$ semana e outro que começa no início do terceiro trimestre e provavelmente termina no segundo ano de vida, coincidindo com o período de menor resistência vascular. Estudos recentes têm indicado associação entre centralização e pós-datismo com oligoâmnio ${ }^{37}$.

Devido ao grande avanço tecnológico na área de diagnóstico médico, freqüentemente o obstetra fica confuso na interpretação dos exames e em sua confiabilidade, muitas vezes tendo dificuldade até na escolha do melhor exame a ser solicitado para um caso específico. A acurácia dos diversos métodos de avaliação de vitalidade fetal é variável em diferentes estudos, estando na dependência de valores de normalidade adotados para os diferentes métodos e também da população estudada. Atualmente existe consenso de que a dopplervelocimetria é o método mais precoce de avaliação do bem-estar fetal.

O diagnóstico de centralização de fluxo sangüíneo fetal está intimamente associado com o sofrimento fetal. Como já relatado na revisão de vários estudos, é um mecanismo de defesa fetal em face da hipoxemia. Portanto, seu diagnóstico deve ser criterioso. Quando informamos ao obstetra que o feto encontra-se centralizado, geramos grande ansiedade não apenas no médico, mas também na paciente e em seus familiares.

Quando o obstetra se depara com um feto "centralizado", deve ter em mente que os resultados perinatais adversos são maiores nestes fetos do que os resultados esperados em relação à prematuridade apenas. Deve, entretanto, considerar criteriosamente as perspectivas de interrupção da gravidez, com a visão voltada para o serviço de neonatologia que deverá receber o concepto, e sua capacidade real de atender crianças que associam freqüentemente prematuridade, restrição prolongada de nutrição e dano variável de suas condições de saúde.

O reconhecimento antenatal de gestação com elevado risco de resultados perinatais adversos é um dos maiores desafios da prática obstétrica. A possibilidade de avaliação qualitativa das condições hemodinâmicas do feto intra-uterino, de forma não-invasiva, representou um grande avanço neste contexto. Neste estudo, os fetos com centralização de fluxo sangüíneo fetal diagnosticado através da dopplervelocimetria, apresentaram alta mortalidade e morbidade, configurando sua utilidade para apoiar decisões clínicas obstétricas.

\section{SUMMARY}

Purpose: evaluation of perinatal outcome of brain-sparing effect detected by color Doppler.

Methods: brain-sparing effect was detected in 32 fetuses at the Ultrasound Service of the Center for Integral Attention to Women's Health at Campinas State University (UNICAMP). The diagnosis of brain-sparing effect was made when the ratio between middle cerebral artery and umbilical artery pulsatility indexes was below one (IPACM/IPAU <1). The measurement was obtained with color Doppler equipment Toshiba SSH-140A.

Results: admission to neonatal intensive care unit (ICU) was necessary in 26 fetuses (89.6\%). The number of days in ICU varied from 1 to 83 days, with a mean of 22 days. Fetal mortality rate was 3 in 32 (9.4\%) and perinatal mortality was 9 in 29 (31\%). Considering the gestational age by the Capurro method, the incidence of birth below 36 weeks was 21 in $32(65.6 \%)$. Intrauterine growth restriction occurred in $71.8 \%$ of the cases and hypoglycemia in $44.8 \%$.

Conclusions: brain-sparing effect is a condition in which the fetus is at serious risk of adverse perinatal outcome and Doppler studies might be helpful in the obstetric management.

KEY WORDS: Doppler velocimetry. Brain-sparing effect. Fetal outcome.

\section{Referências}

1. Brennan SC, McLaughlin MK, Chez RA. Effects of prolonged infusion of beta-adrenergic agonists on uterine and umbilical blood flow in pregnant sleep. Am J Obstet Gynecol 1977; 128:709-15.

2. Clapp JF $3^{\text {rd }}$. The relationship between blood flow and oxygen uptake in the uterine and umbilical circulations. Am J Obstet Gynecol 1978; 132:410-3.

3. Metcalfe J, Romney SL, Ramsey LH, Reide DE, Burwell CS. Estimation of uterine blood flow in normal human pregnancy at term. $\mathrm{J}$ Clin Invest $1955 ; 34: 1632-8$.

4. Moll W, Kunzel W, Herberger J. Hemodynamic implications of hemochorial placentation. Eur J Obstet Gynecol Reprod Biol 1975; 5:67-74.

5. FitzGerald DE, Drumm JE. Non-invasive measurement of human fetal circulation using ultrasound: a new method. Br Med J 1977, 2:1450-1.

6. Maulik D, Saini VD, Nanda NC, Rosenzweig MS. Doppler evaluation of fetal hemodynamics. Ultrasound Med Biol 1982; 8:705-10.

7. Pourcelot L. Applications cliniques de l'examen Doppler transcutané. In: Peronneau P, éditeur. Vélocimetrie Ultrasonore Doppler. Paris: Inserm; 
1974. p.213-40.

8. Gosling RG, King DH. Ultrasound angiology. In: Marcus AW, Adamson J, editors. Arteries and Veins. $1^{\text {st }}$ ed. Edinburg: Churchill-Livingstone; 1975. p.61-71.

9. Stuart B, Drumm J, Fitzgerald DE, Duignan NM. Fetal blood velocity waveforms in normal pregnancy. Br J Obstet Gynaecol 1980; 87:780-5.

10.Schulman H, Fleischer A, Stern W, Farmakides G, Jagani N, Blattner P. Umbilical velocity wave ratios in human pregnancy. Am J Obstet Gynecol 1984; 148:985-90.

11.Cohen-Overbeek T, Pearce JM, Campbell S. The antenatal assessment of utero-placental and fetoplacental blood flow using Doppler ultrasound. Ultrasound Med Biol 1985; 11:329-39.

12.Trudinger BJ, Giles WB, Cook CM, Bombardieri J, Collins L. Fetal umbilical artery flow velocity waveforms and placental resistance: clinical significance. Br J Obstet Gynaecol 1985; 92:2330 .

13.Saling E. El Niño deste el punto de vista obstétrico. $1^{\mathrm{a}}$ ed. Barcelona: Cientifica Médica; 1969. p.167.

14.Cohn HE, Sacks EJ, Heymann MA, Rudolph AM. Cardiovascular responses to hypoxemia and acidemia in fetal lambs. Am J Obstet Gynecol $1974 ; 15: 817-24$.

15.Peeters LL, Sheldon RE, Jones MD Jr, Makowski EL, Meschia G. Blood flow to fetal organs as a function of arterial oxygen content. Am J Obstet Gynecol 1979; 135:637-46.

16.Wladimiroff JW, Tonge HM, Stewart PA. Doppler ultrasound assessment of cerebral blood flow in the human fetus. Br J Obstet Gynaecol 1986; 93:471-5.

17.Arduini D, Rizzo G. Prediction of fetal outcome in small for gestational age fetuses: comparison of Doppler measurements obtained from different fetal vessels. J Perinat Med 1992; 20:29-38.

18.Gramellini D, Folli MC, Raboni S, Vadora E, Merialdi A. Cerebral-umbilical Doppler ratio as a predictor of adverse perinatal outcome. Obstet Gynecol 1992; 79:416-20.

19.Arias F. Accuracy of the middle-cerebral-to-umbilicalartery resistance index ratio in the prediction of neonatal outcome in patients at high risk for fetal and neonatal complications. Am J Obstet Gynecol 1994; 171:1541-5.

20.Akalin-Sel T, Nicolaides KH, Peacock J, Campbell S. Doppler dynamics and their complex interrelation with fetal oxygen pressure, carbon dioxide pressure, and $\mathrm{pH}$ in growth-retarded fetuses. Obstet Gynecol 1994; 84:439-44.

21.Bahado-Singh RO, Kovanci E, Jeffres A, et al. The Doppler cerebroplacental ratio and perinatal outcome in intrauterine growth restriction. Am J Obstet Gynecol 1999; 180:750-6.
22.Apgar V. A proposal for a new method of evaluation of the newborn infant. Anesth Analg 1953; $32: 260-7$

23. Capurro H, Konichezky S, Fonseca D, CaldeyroBarcia R. A simplified method for diagnosis of gestational age in the newborn infant. J Pediatr 1978; 93:120-2.

24.Franzin CMMO. Avaliação ultra-sonográfica da idade gestacional através da biometria fetal - estudo longitudinal [dissertação]. Campinas: Universidade Estadual de Campinas; 1993.

25.Hadlock FP, Harrist RB, Martinez-Poyer J. In utero analysis of fetal growth: a sonographic weight standard. Radiology 1991; 181:129-33.

26.Lubchenco LO, Hansman C, Boyd E, Dressler M. Intrauterine growth as estimated from liveborn birth-weight data at 24 to 42 weeks of gestation. Pediatrics 1963; 32:793-800.

27.Cerri GG, Mólnar LJ, Vezozzo DCP. Doppler. São Paulo: Sarvier; 1996. Aplicação do Doppler em obstetrícia. p.185-219.

28.Arduini D, Rizzo G. Normal values of pulsatility index from fetal vessels: a cross-sectional study on 1556 healthy fetuses. J Perinat Med 1990; 18:165-72.

29. Mari G, Deter RL. Middle cerebral artery flow velocity waveforms in normal and small-for-gestationalage fetuses. Am J Obstet Gynecol 1992; 166:1262-70.

30.Franzin CMMO, Silva JLP, Mezzalira R, Surita F, Marussi EF. Componente distólico ausente na artéria umbilical: análise de 30 casos. Rev Bras Ginecol Obstet 1996; 18:491-4.

31.Freeman JM, Nelson KB. Intrapartum asphyxia and cerebral palsy. Pediatrics 1988; 82:240-9.

32.Sykes GS, Molloy PM, Johnson P, et al. Do Apgar scores indicate asphyxia? Lancet 1982; 27:494-6.

33. Montenegro CAB, Rezende Filho J, Silva LGP. Centralização fetal. Femina 1994; 22:203-15.

34.Dawes GS, Lewis BV, Milligan JE, Roach MR, Talner NS. Vasomotor responses in the hind limbs of foetal and newborn lambs to asphyxia and aortic chemoreceptor stimulation. J Physiol 1968; 195:55-81.

35.Giles WB, Trudinger BJ, Baird PJ. Fetal umbilical artery flow velocity waveforms and placental resistance: pathological correlation. $\mathrm{Br} \mathrm{J}$ Obstet Gynaecol 1985; 92:31-8.

36.Dobbing J, Sands J. Timing of neuroblast multiplication in developing human brain. Nature 1970; 226:639-40.

37.Selam B, Koksal R, Ozcan T. Fetal arterial and venous Doppler parameters in the interpretation of oligohydramnios in postterm pregnancies. Ultrasound Obstet Gynecol 2000; 15:403-6. 